\title{
Modeling marine mercury cycling - a discussion of current capabilities, uncertainties and limitations
}

\author{
JOHANNES BIESER ${ }^{1}$, UTE DAEWEL ${ }^{2}$, JOACHIM KUSS ${ }^{3}$ \\ AND CORINNA SCHRUM ${ }^{4}$
}

${ }^{1}$ Helmholtz-Zentrum Hereon

${ }^{2}$ Helmholtz-Zentrum Geesthacht

${ }^{3}$ Leibniz Institute for Baltic Sea Research Warnemünde

${ }^{4}$ Helmholtz-Zentrum Geesthacht Centre for Materials and Coastal Research

Presenting Author: Johannes.Bieser@hzg.de

For the effectiveness evaluation of the Minamata Convention, a key question is to understand the impact of external drivers (e.g. anthropogenic emission reductions, climate change) on methylmercury accumulation in sea food. Thus, in recent years the development and improvement of marine $\mathrm{Hg}$ models has become a pressing issue. We argue that before we can use such models to project the fate of $\mathrm{Hg}$ in the marine environment it is necessary to determine and understand the predictability of mercury species. This knowledge is well established for atmospheric $\mathrm{Hg}$ models but currently lacking for models of $\mathrm{Hg}$ in water and biota. Moreover, detailed studies of $\mathrm{Hg}$ cycling are deemed necessary to develop simplified and thus more performant mechanisms for the use in global models.

Here, we show results of the high-resolution ocean-ecosystemchemistry model MECOSMO. MECOSMO is the first model to explicitly resolve all known processes relevant to marine mercury cycling with resolutions of $1 \mathrm{~km}$ to $100 \mathrm{~km}$. With the rigor usually applied to 'classical' pollutants in atmospheric chemistry transport models such as ozone and particulate matter, we investigate the capability to reproduce observed concentrations for all relevant mercury species (including shortand long-term variability). We evaluate the capabilities and limitations of models based on the current knowledge of marine $\mathrm{Hg}$ cycling. Using different chemical mechanisms, reaction rates, and parametrizations we identify the main sources for uncertainty in the model with the aim to inspire future observational studies to address these issues and supply central parameters needed to improve marine $\mathrm{Hg}$ models.

This work is part of the MSCA ITN GMOS-TRAIN network which facilitates close collaboration between analytical and modling research groups. 